L. Barbanti, IME-USP, CP. 66281, 05315-950 S. Paulo (SP) - Brasil. e-mail: barbanti@ime.usp.br

\title{
SIMPLY REGULATED FUNCTIONS AND SEMIVARIATION IN UNIFORMLY CONVEX SPACES
}

\begin{abstract}
In the last two decades the Riemann generalized integral, having values in Banach spaces, has been increasingly studied.

The development in this area concerns mainly with the HenstockKurzweil (see e.g. [1], [2]), and the Dushnik and the Young integrals (see e.g. [3]). Recently it has appeared in the literature many proper applications in this field (proper here considered in the sense that the results are not disguises of an essentially finite dimensional frame) (see e.g. [4], [5]).

In this paper we give a result having its roots in this frame.
\end{abstract}

\section{Bilinear Triples, Regulated Functions and Bounded Variation}

Consider the Banach spaces $X, Y$ and $Z$, and suppose the existence of a bilinear mapping $B: X \times Y \rightarrow Z$ satisfying

$$
\|B(x, y)\|_{Z} \leq\|x\|_{X} \cdot\|y\|_{Y} .
$$

A triple of spaces with a bilinear mapping having such property is named a bilinear triple and we will denote this fact by $\mathcal{B}=(X, Y, Z)$.

Key Words: bilinear triples, generalized Riemann integral, simply regulated functions, semivariation

Mathematical Reviews subject classification: 47XX, 26A39

Received by the editors May 12, 1997 
Let $[a, b] \subset \mathbb{R}$ and $D$ the set of all finite partitions $d=\left\{t_{0}=a<t_{1}<\right.$ $\left.\cdots<t_{|d|}=b\right\}$. We say that $x:[a, b] \rightarrow X$ is of bounded variation on $[a, b]$ if,

$$
\operatorname{var}(x)=\sup _{d \in D}\left\{\sum_{j=1}^{|d|}\left\|x\left(t_{j}\right)-x\left(t_{j-1}\right)\right\|\right\}<\infty .
$$

The set of all mappings $x:[a, b] \rightarrow X$ with $\operatorname{var}(x)<\infty$ will be denoted by $B V([a, b], X)$.

The function $x:[a, b] \rightarrow X$ is said to be regulated on $[a, b]$ if $x$ has only discontinuities of the first kind.

The set of all regulated functions $x:[a, b] \rightarrow X$ will be denoted by $G([a, b], X)$, and is a Banach space when endowed with the sup norm.

Let be $\mathcal{B}=(X, Y, Z)$ a bilinear triple. The mapping $x:[a, b] \rightarrow X$ is said to be $\mathcal{B}$-regulated (or sometimes simply regulated (see $[3]$ )) on $[a, b]$ if for every $y \in Y$ with $\|y\|_{Y} \leq 1$, the mapping $x y:[a, b] \rightarrow Z$, defined by

$$
t \longmapsto x(t) y \in Z, \quad t \in[a, b]
$$

is regulated. We will denote the set of such function by $(\mathcal{B}) G([a, b], X)$.

Finally, let us define for all $d \in D$,

$$
V(x, d)=\sup \left\{\left\|\sum_{j=1}^{|d|}\left[x\left(t_{j}\right)-x\left(t_{j-1}\right)\right] y_{j}\right\|_{Z} ; y_{j} \in Y \text { and }\left\|y_{j}\right\| l_{Y} \leq 1\right\}
$$

and $(\mathcal{B}) \operatorname{var}(x)=\sup _{d \in D} V(x, d)$.

A mapping $x:[a, b] \rightarrow X$ satisfying $(\mathcal{B}) \operatorname{var}(x)<\infty$ is said to be of $(\mathcal{B})$-bounded variation (or sometimes of bounded semivariation (see e.g. [3])) on $[a, b]$. The set of such mappings will be denoted by $(\mathcal{B}) B V([a, b], X)$.

For more details on bilinear triples, see e.g. [2] and [6].

\section{Riemann-Stieltjes Type Integrals}

\subsection{The question}

Let us consider from now $\mathcal{B}=(L(X), X, X)$, where $L(X)$ denotes the set of all linear bounded operators on $X$.

Let the (generalized) Riemann-Stieltjes integral on $[a, b]$ be denoted by

$$
F_{K}[x](t)=\odot \int_{a}^{t} d[K(s)] x(s), \quad(a \leq t \leq b),
$$


where $x \in G([a, b], X)$ and such that in $\odot$ either the Henstock-Kurzweil or the Dushnik or the Young integrals are being considered.

It is well known that $F_{K}$ is an operator on $G([a, b], X)$ if:

1. considering in $\odot$ either the Dushnik or Young integral:

$$
K \in(\mathcal{B}) B V([a, b], L(X))
$$

(see [3, Th. 1.10 and Th. 1.16]).

2. considering in $\odot$ the Henstock-Kurzweil integral:

$$
K \in(\mathcal{B}) B V([a, b], L(X)) \cap(\mathcal{B}) G([a, b], L(X))
$$

(see [2, Prop. 15]).

Now, looking at these two statements it is possible to ask in a quite natural way: is it really necessary to have $K \in(\mathcal{B}) G([a, b], L(X))$ in the HenstockKurzweil case?

Actually it is possible to work a little about an answer to this question:

\subsection{An Answer}

We say that a Banach space $X$ is uniformly convex if for every $0<\varepsilon \leq 2$ there exists an $\delta(\varepsilon)>0$ such that: if $x, y \in X$, with $\|x\|=\|y\|=1$ and $\|x-y\|>\varepsilon$, then $\|x+y\| \leq 2(1-\delta(\varepsilon))$.

A large class of spaces are uniformly convex, among them the Hilbert ones.

If $X$ is a uniformly convex space, then there exists a norm, $\|\cdot\|$, equivalent to the original one, satisfying: there are $p>2$ and $\delta>0$ such that for every $x, y \in X:$

$$
\|x\|^{p}+\delta\|y\|^{p} \leq \frac{\|x+y\|^{p}}{2}+\frac{\|x-y\|^{p}}{2} .
$$

The property (UC) can be replaced by the equivalent one:

(A) either $\|x+y\|^{p} \geq\|x\|^{p}+\delta\|y\|^{p} \quad$ or $\quad\|x-y\|^{p} \geq\|x\|^{p}+\delta\|y\|^{p}$.

Observe that if $X$ is a Hilbert space then (A) is satisfied with $p=2$ and $\delta=1$. For more details concerning the theory of uniformly convex spaces see e.g. [7].

Now, it is possible in this framework, to state our main result: 
Theorem 1. Theorem 1 If $X$ is a uniformly convex space then

$$
(\mathcal{B}) B V([a, b], L(X)) \subset(\mathcal{B}) G([a, b], L(X)) \text {. }
$$

Proof. Suppose $\varphi \notin(\mathcal{B}) G([a, b], L(X))$. Then, there exists an $y_{0} \in X, 0<$ $\left\|y_{0}\right\| \leq 1$, with $\varphi_{y_{0}}$ (defined by $\left.\varphi_{y_{0}}(t)=\varphi(t) y_{0}\right)$ having no - say, left lateral limit at a point $\bar{t} \in(a, b]$. Hence - following the Cauchy convergence criterion - we get an $\varepsilon_{0}>0$, and a sequence $\left\{t_{n}\right\}$ in $[a, b]$, with $t_{n} \uparrow \bar{t}$, in such a way that:

(P) $\quad \forall k \in \mathbb{N}, \exists m_{k}>n_{k}>k$ with $\left\|\varphi_{y_{0}}\left(t_{m_{k}}\right)-\varphi_{y_{0}}\left(t_{n_{k}}\right)\right\| \geq \varepsilon_{0}$.

Applying successively (P) for $k=k_{1}=1, \ldots, k_{p}=m_{k_{p-1}}+1, \ldots,(p \geq 2)$, we finally get: there exists a sequence of intervals $\left[t_{1}^{j}, t_{2}^{j}\right]$ with $\left[t_{1}^{j+1}, t_{2}^{j+1}\right] \subset$ $\left(t_{2}^{j}, \bar{t}\right), j=1,2, \ldots$, satisfying

$$
\left\|\varphi_{y_{0}}\left(t_{1}^{j}\right)-\varphi_{y_{0}}\left(t_{2}^{j}\right)\right\| \geq \varepsilon_{0} .
$$

Now, taking the following elements in $D$ :

$$
d_{n}=\left\{a, t_{1}^{1}, t_{2}^{1}, t_{1}^{2}, t_{2}^{2}, \ldots, t_{1}^{n}, t_{2}^{n}, b\right\}, \quad(n \geq 1)
$$

it is possible to prove in a recurrent way that

$$
V\left(\varphi, d_{n}\right) \geq[1+(n-1) \delta]^{1 / p} \cdot \varepsilon_{0} .
$$

Indeed: if $n=1$ then

$$
\left\|\varphi_{y_{0}}\left(t_{1}^{1}\right)-\varphi_{y_{0}}\left(t_{2}^{1}\right)\right\| \geq \varepsilon_{0} .
$$

Suppose $n \geq 1$. Let be

$$
s_{n}=\sum_{j=1}^{n}\left[\varphi_{y_{0}}\left(t_{1}^{j}\right)-\varphi_{y_{0}}\left(t_{2}^{j}\right)\right],
$$

and consider the hypothesis: $\left\|s_{n}\right\| \geq[1+(n-1) \delta]^{1 / p} \cdot \varepsilon_{0}$. Then by choosing either $y_{0}$ or $-y_{0}$ in accordance with $(\mathrm{A})$, we have

$$
\left\|s_{n+1}\right\|^{p} \geq\left\|s_{n}\right\|^{p}+\delta\left\|\left[\varphi\left(t_{1}^{n+1}\right)-\varphi\left(t_{2}^{n+1}\right)\right] \cdot y_{0}\right\|^{p} .
$$

Hence,

$$
\left\|s_{n+1}\right\|^{p} \geq\left\|s_{n}\right\|^{p}+\delta \varepsilon_{0}^{p} \geq(1+n \delta) \cdot \varepsilon_{0}^{p} .
$$


Observe that if we complete the sum $s_{n}$ - calling $a=t_{2}^{0}$ - with

$$
\left[\varphi\left(t_{2}^{i-1}\right)-\varphi\left(t_{1}^{i}\right)\right] \cdot 0=\left[\varphi\left(t_{2}^{n}\right)-\varphi(b)\right] \cdot 0=0, \quad(1 \leq i \leq n),
$$

we get for every $n \geq 1$,

$$
V\left(\varphi, d_{n}\right) \geq\left\|s_{n}\right\|
$$

and consequently the result $(T)$.

So, $\varphi \notin(\mathcal{B}) B V([a, b], L(X))$.

\section{References}

[1] J. Kurzweil, Nichtabsolut Konvergente Integrale, BBS B.G. Teubner Verlagsg., Leipzig, 1980.

[2] Š. Schwabik, Abstract Perron - Stieltjes integral, Preprint, Ak. Ved, Praha, 1996.

[3] C. S. Hönig, Equations integràles generalisées et applications, Pub. Math. d'Orsay 5, 1983.

[4] O. Diekmann et all, Perturbing semi-groups by solving Stieltjes renewal equations, Diff. and Integral Eq. v. 6, n. 1 (1993), 155-181.

[5] O. Diekmann et all, Perturbing evolutionary systems by step responses and cumulative outputs, Diff. and Integral Eq. v. 8, n. 5 (1995), 1205-1244.

[6] C. S. Hönig, Volterra Stieltjes integral equations, Math. Studies 16, N. Holland, Publ. Co., 1975.

[7] D. Kutzarova and B. Lin, Locally uniformly convex Banach spaces, Math. Balkanica n. 2-3 (1994), 203-210, 
L. BARBANTI 